\title{
Colorization of Gray Scale Images in YCbCr Color Space Using Texture Extraction and Luminance Mapping
}

\author{
Mrs. Smriti Kumar ${ }^{1}$, Mr. Deepak Singh ${ }^{2}$ \\ ${ }^{1,2}$ (Department of Electronics and Telecommunication, Chhatrapati Shivaji Institute of Technology, India)
}

\begin{abstract}
Gray scale image colorization is a useful application in the world of image processing. This work presents a general technique for "colorizing" grayscale images by transferring color between a source or color image and a destination or target or grayscale image by matching luminance and texture information between the images. This technique of the gray image coloring uses a very simple algorithm that chooses a decorrelated color space and then applies simple operations there. Thus the color space with decorrelated axes is a useful tool for manipulating color images in this work. Colorization of a gray scale image is achieved here by matching texture features of gray image with texture features of windows of colored image and then by imposing mean and standard deviation onto the data points in a simple operation, thus believable output images are obtained given suitable input images. Here, we use texture features like energy, entropy, homogeneity, contrast and correlation based on correlation matrix for the purpose of texture matching between two images.
\end{abstract}

Keywords: Colorization, Contrast, Correlation, Energy, Entropy, Homogeneity, Mean and Standard Deviation, YCbCr Color Space.

\section{INTRODUCTION}

Color can increase the visual appeal of grayscale images such as old black and white photos, classic movies or scientific outcomes, some examples are image modalities in medicine, which only acquire grayscale images such Magnetic Resonance Imaging (MRI), X-ray and Computerized Tomography (CT) images can be enhanced with color for presentations and demonstrations. In addition, the information content of some scientific images can be perceptually enhanced with color by exploiting texture, chromaticity as well as luminance variations.

Adding colors to a grayscale image actually involves assigning three-dimensional (RGB) pixel values to an image which varies along only one dimension (luminance or intensity). In fact, different colors may have the same luminance value but may vary in hue or saturation, hence, the problem of colorizing grayscale images has no inherently "correct" solution. Due to these reasons, human interaction usually plays a large role in the colorization process. Term 'Colorization' was introduced in 1970 and was later patented by Wilson Markle. Colorization trends have been classified into three categories - Hand coloring, Semi automatic coloring, and automatic coloring. Semary N. A. [7]. Hand coloring has been long used by artists as a way of showing their talent. Usually image editing software's like Adobe Photoshop or Paint shop Pro are used similarly to convert gray images to color images. Semiautomatic coloring refers to mapping luminance values to color values, which is done by converting the gray image to an indexed image. Pseudo coloring is a common example [4][5]. Automatic coloring can be classified into three categories according to the source of colors to be transferred to the gray image pixels, [7] which are transformational coloring, Image Matching and User Selection. Technique that is mentioned here belongs to the category of Image matching/ Coloring by Reference. It relies on the fact that for each gray pixel $I_{g}(x, y)$ in target gray scale image there exists a colored pixel $I_{s}\left(x_{2}, y_{2}\right)$ in reference or source image such that the distance (some similarity measure) between them is the minimum value. The chromatic values in " $\mathrm{I}_{\mathrm{s}}$ " are transferred to " $\mathrm{I}_{\mathrm{g}}$ " and the achromatic value of " $\mathrm{I}_{\mathrm{g}}$ " remains. Here, the source colored image need not be the colored version of gray scale image, it can be any similar image in RGB. When a typical three channel image is represented in the RGB color space, correlation exists between the different channels' values, thus if we change the appearance of a pixel's color in a coherent way, we must modify all color channels in tandem. Hence, any color modification process gets complicated. So, an orthogonal color space which doesn't have correlations between the axes is the foremost requirement.

Ruderman et al [1] developed a color space, called laß, based on data-driven human perception research that assumes that the human visual system is ideally suited for processing natural scenes. There's little correlation between the axes in la $\beta$ space, which allows application of different operations in different color 
channels without causing cross-channel artifacts. Apart from this, $l \alpha \beta$ color space is logarithmic, which implies to a first approximation that uniform changes in channel intensity tend to be equally detectable [6]. This makes $l \alpha \beta$ color space a very popular choice for gray image colorization.

We have chosen another decorrelated color space $\mathrm{YCbCr}$ for colorization purpose. $\mathrm{YCbCr}$ color space also provides three decorrelated channels $\mathrm{Y}, \mathrm{Cb}$ and $\mathrm{Cr}$. Channel $\mathrm{Y}$ is achromatic luminance channel, where as chromatic channels $\mathrm{Cb}$ and $\mathrm{Cr}$ correspond to the difference between blue component with a reference value and difference between red components with a reference value, respectively. This space also allows selective transfer of chromatic $\mathrm{Cb}$ and $\mathrm{Cr}$ channels from color image to grayscale image without introducing crosschannel artifacts.

For colorization purpose we convert our source image into a decorrelated color space and then divide this image into small windows, whose size may vary according to the type of image. After this we extract texture features like energy, entropy, homogeneity, contrast and correlation based on correlation matrix for the purpose of texture matching, for each window of source image as well as for the target grayscale image. Then we compare each window of the source (colored) image with target image and find out the best matching window in source image based on texture feature matching. Then we compare the data points in this best matching source window by computing mean and standard deviations along each of the three axes of the sample data points and the data points in target image, in $\mathrm{YCbCr}$ color space. Then, we scale the data points by factors determined by the respective standard deviations. Then the chromatic values of the best matching source image window's data points are transferred to the target image pixel. The resulting image of this process is colorized version of target gray scale image. All the coding for above processing has been done in MATLAB 2010 version.

\section{METHODOLOGY}

The basic method here matches the three-dimensional distribution of texture features and color values between the images and then transforms the color distribution of the source (colored) image to match the distribution of the target (grayscale) image. A grayscale image is represented by a one dimensional distribution, hence only the luminance channels can be matched between the two images. Apart from this we also match texture features like energy, entropy, homogeneity, contrast and correlation based on correlation matrix. Once the target image matches a source image window, the color information is transferred between pixels but the original luminance value is retained. Our goal is to manipulate RGB images, which are often of unknown phosphor chromaticity; a very popular way is to convert RGB signals to Ruderman et al.'s [1] perception-based color space la $\beta$. We have converted the gray scale and reference colored image to $\mathrm{YCbCr}$ color space, for processing the images in this color space for colorization of gray scale image.

To show the significance of choosing the right color space, we compared three different color spaces. The color spaces chosen here are RGB as used by most graphics algorithms, CIECAM97s, $l \alpha \beta$ and YCbCr. We chose the CIECAM97s color space because it is closely related to the $l \alpha \beta$ color space. Its transformation matrix to convert from RGB to CIECAM97s is:

$$
\begin{aligned}
& {\left[\begin{array}{c}
L \\
M \\
S
\end{array}\right]=\left[\begin{array}{lll}
.3811 & .5783 & .0402 \\
.1967 & .7244 & .0782 \\
.0241 & 0.1288 & .8444
\end{array}\right]\left[\begin{array}{l}
R \\
G \\
B
\end{array}\right],} \\
& {\left[\begin{array}{c}
A \\
C 1 \\
C 2
\end{array}\right]=\left[\begin{array}{ccc}
2.00 & 1.00 & 0.05 \\
1.00 & -1.09 & 0.09 \\
0.11 & 0.11 & -0.22
\end{array}\right]\left[\begin{array}{c}
L \\
M \\
S
\end{array}\right],}
\end{aligned}
$$

The two chromatic channels $C 1$ and $C 2$ resemble the chromatic channels in $l \alpha \beta$ space, except a scaling of the axes. The achromatic channel is different (see Fig. 2). Another difference is that CIECAM97s operates in linear space, and $l \alpha \beta$ are defined in log space.

Using image in Fig.1, we produced scatter plots of 2,000 randomly chosen data points in $l \alpha \beta, \mathrm{YCbCr}$, RGB, and CIECAM97s. Fig.2 depicts these scatter plots that show three pairs of axes plotted against each other. The data points are decorrelated if the data are axis aligned, which is the case for all three pairs of axes in all $\mathrm{YCbCr}, l \alpha \beta$ and CIECAM97s spaces. The RGB color space shows almost complete correlation between all pairs of axes because the data cluster around a line with a 45 -degree slope. This provides some validation for Ruderman et al.'s color space $l \alpha \beta[1]$ as well as for $\mathrm{YCbCr}$ which we have used in this work.

In order to transfer chromaticity values from the source to the target, first of all, the source image is divided into small windows of equal size and then each window in the source image is matched to the gray scale image based on texture features of that window. We calculate texture features like energy, entropy, homogeneity, contrast and correlation based on correlation matrix based on following formulae [2]: 


$$
\begin{aligned}
& S=-\sum_{i} \sum_{j} P[i, j] \log P[i, j], \\
& J=-\sum_{i} \sum_{j} P^{2}[i, j], \\
& \text { Con }=-\sum_{i} \sum_{j}(i-j)^{2} P[i, j], \\
& H=-\sum_{i} \sum_{j} P[i, j] /(1+|i-j|)^{\prime} \\
& \text { Cor } \left.=-\frac{1}{\sigma_{i} \sigma_{j}}\left[\sum_{i} \sum_{j} i j P[i, j]-\operatorname{qi}_{i}\right]_{j}\right],
\end{aligned}
$$

Where $\mathrm{S}$ is entropy, $\mathrm{J}$ is energy, Con is contrast, $\mathrm{H}$ is homogeneity, Cor is correlation. $\mathrm{P}[\mathrm{i}, \mathrm{j}]$ is the value of element in co-occurrence matrix and $[\mathrm{i}, \mathrm{j}]$ is the coordinate, $\mu_{\mathrm{i}}$ and $\mu_{\mathrm{j}}$ are mean of $\mathrm{p}_{\mathrm{i}}$ and $\mathrm{p}_{\mathrm{j}},[2]$ respectively, where :

$p_{i}=-\sum_{j} P[i, j]$,
$p_{j}=-\sum_{i} P[i, j]$,

$\mathrm{W}(\mathrm{i}, \mathrm{j})=e^{-d\left(x_{i}-x_{j}\right) / 2 \omega^{2}}$,

texture features are weighted appropriately using following formulae [9]:

Where $\omega$ is the parameter under consideration and:

$d\left(x_{i}-x_{j}\right)=|| x_{i}-x_{j}||^{2}$,

Thus texture similarity measures are weighted properly and a texture similarity measure 'TM' based

on Euclidean distance is calculated for each window in the source image and for target image as well [2]. Based on this texture similarity measure each of the windows in source image is compared with the target image and the best matching window is thus found out.

For colorization purpose, the mean and standard deviations of the data points along each of the three axes are required and their luminance value is determined by the $\mathrm{Y}$ channel in $\mathrm{YCbCr}$ space. Hence, we compute these measures for both the best matching source window and for the target image, for every pixel. We compute the means and standard deviations for each axis separately in $\mathrm{YCbCr}$ space. First, we subtract the mean from the data points [8] [10]:

$\begin{aligned} Y^{*} & =Y-\langle Y\rangle, \\ C b^{*} & =C b-\langle C b\rangle, \\ C r^{*} & =C r-\langle C r\rangle\end{aligned}$

$Y^{\prime}=\frac{\sigma_{t}^{Y}}{\sigma_{s}^{Y}} Y^{*}$,

$C b^{\prime}=\frac{\sigma_{t}^{C b}}{\sigma_{s}^{C b}} C b^{*}$,

$C r^{\prime}=\frac{\sigma_{t}^{C r}}{\sigma_{s}^{C r}} C r^{*}$,

Using above formulae pixel by pixel comparison is done between the best matching source image window and in the target image. The best matching pixel in source window to a pixel in target image is thus found out and its chromatic values of are transferred to the corresponding pixel of target image window, but their luminosity values are retained. This is done pixel by pixel for target image and the result obtained thus is the colorized version of target gray scale image.

We intend here to transfer one image's appearance to another; hence it's possible to select source and target images that may not work well together, but the result's quality depends on the images' similarity in composition. The results are shown in subsequent figures.

We have also compared the resulting images obtained based on time required for processing, Mean Square Error (MSE), Peak Signal to Noise Ratio(PSNR), Normalized Cross correlation(NK), Structural Content(SC), Maximum Difference(MD), Normalized Absolute Error(NAE), Visual Quality with respect to the target gray scale image. This calculation is based on following formulae [3]: 
$M S E=\frac{1}{M N} \sum_{j=1}^{M} \sum_{k=1}^{N}\left(x_{j . k}-x_{j, k}^{\prime}\right)^{2}$,

$\operatorname{PSNR}=10 \operatorname{Yog} \frac{\left(2^{n}-1\right)^{2}}{M S E}$,

$N K=\sum_{j=1}^{M} \sum_{k=1}^{N}\left(x_{j . k} x_{j, k}^{\prime}\right)^{1} / \sum_{j=1}^{M} \sum_{k=1}^{N}\left(x_{j . k}\right)^{2}$,

$S C=\frac{\sum_{j=1}^{M} \sum_{k=1}^{N}\left(x_{j . k}\right)^{2}}{\sum_{j=1}^{M} \sum_{k=1}^{N}\left(x_{j, k}^{\prime}\right)^{2}}$,

$M D=\operatorname{Max}\left(\left|x_{j . k}-x_{j, k}^{\prime}\right|\right)$

$N A E=\frac{\sum_{j=1}^{M} \sum_{k=1}^{N}\left|x_{j . k-} x_{j, k}^{\prime}\right|}{\sum_{j=1}^{M} \sum_{k=1}^{N}\left|x_{j . k}\right|}$

The results are tabulated in Table 1 and 2.

\section{RESULT}

Our colorization method produces believable colorized output images, as is obvious from the results in Fig. $(3-10)$. We have colorized images in various color spaces and our method produced best results in $\mathrm{YCbCr}$ color space, we have presented a comparison between results obtained by colorization in $\mathrm{YCbCr}$ color space using our method and a very popular technique for colorization in $l \alpha \beta$ color space [8][10] in Table (1-2). The results are compared with the original target image based on various parameters like Time Taken, Mean Square Error (MSE), Peak Signal to Noise Ratio (PSNR), Normalized Correlation (NK), Maximum Difference (MD), Normalized Absolute Error (NAE) and Visual Quality Obtained. As is apparent from this comparison colorization in $\mathrm{YCbCr}$ color space produces much better results.

Images of various dimensions ranging from 256 X256 to $1024 \times 1024$ were tested and the method proved successful, the time taken for colorization ranges from 25 seconds to 275 seconds.

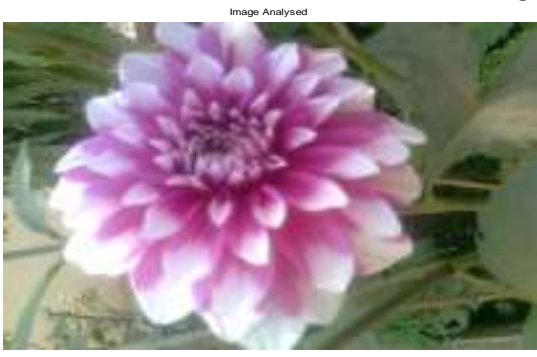

Figure1. Image Analyzed

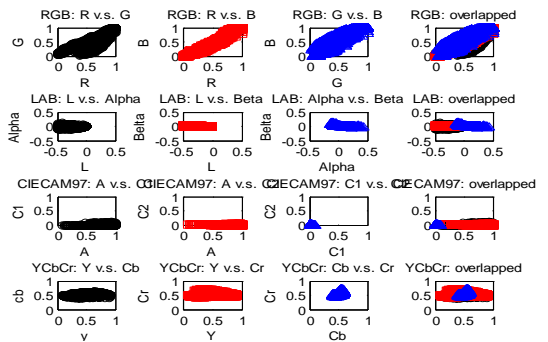

Figure 2. Comparisons between various color spaces of Image Analyzed

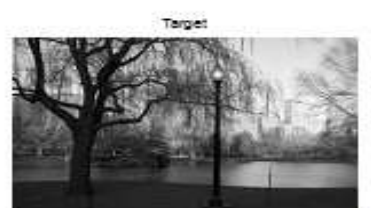

Figure3. Gray Scale Target Image ( $275 \mathrm{X}$ 183)

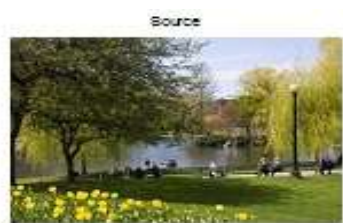

Figure4. Colored Source Image

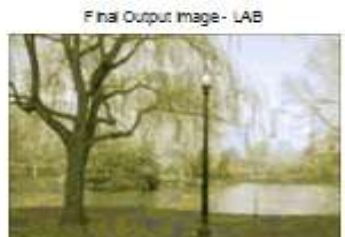

Figure5. Target Image Colored by ConventionalMethod

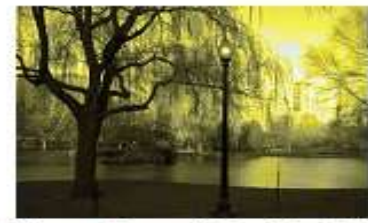

Figure6. Target Image Colored by Proposed Method 


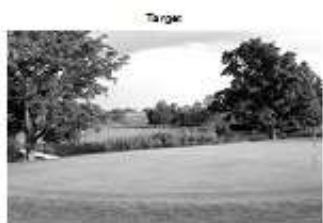

Figure7. Gray Scale Target Image ( 259 X 194) Fins Cutout image

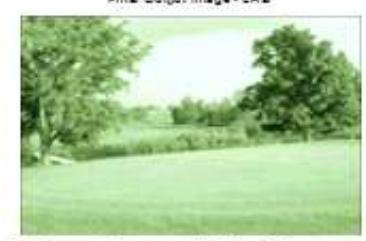

Figure9. Target Image Colored by ConventionalMethod

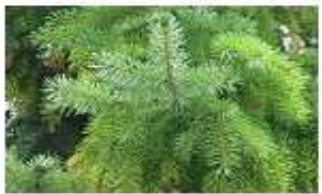

Figure8. Colored Source Image

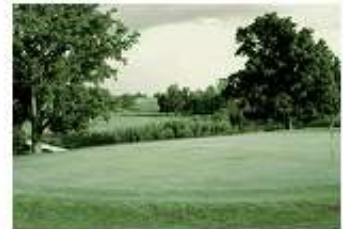

Figurel0. Target Image Colored by Proposed

Table 1 Comparison between results of Fig. 5 and Fig. 6

\begin{tabular}{|c|c|c|c|c|c|c|c|c|}
\hline Parameters $\rightarrow$ & $\begin{array}{c}\text { TIME } \\
\text { TAKEN } \\
\text { Different } \\
\text { methods }\end{array}$ & MSE & PSNR & NK & SC & MD & NAE & $\begin{array}{c}\text { VISUAL } \\
\text { QUALITY }\end{array}$ \\
\hline $\begin{array}{c}\text { Proposed } \\
\text { Technique }\end{array}$ & 4.38 & 341.6446 & 22.7951 & 0.8459 & 1.3884 & 19 & 0.1845 & GOOD \\
\hline $\begin{array}{c}\text { Conventional } \\
\text { Technique }\end{array}$ & 27.0142 & 3249.8 & 13.0123 & 1.3706 & 0.4971 & 114 & 0.5329 & AVERAGE \\
\hline
\end{tabular}

Table 2 Comparisons between results of Fig. 9 and Fig. 10

\begin{tabular}{|c|c|c|c|c|c|c|c|c|}
\hline \multicolumn{1}{|c|}{$\begin{array}{c}\text { Parameters } \rightarrow \\
\text { Different } \\
\text { methods } \downarrow\end{array}$} & $\begin{array}{c}\text { TIME } \\
\text { TAKEN } \\
\text { (SEC) }\end{array}$ & MSE & PSNR & NK & SC & MD & NAE & $\begin{array}{c}\text { VISUAL } \\
\text { QUALITY }\end{array}$ \\
\cline { 1 - 6 } $\begin{array}{c}\text { Proposed } \\
\text { Technique }\end{array}$ & 14.55 & 334.192 & 22.8908 & 0.8950 & 1.2447 & 19 & 0.1281 & GOOD \\
\hline $\begin{array}{c}\text { Conventional } \\
\text { Technique }\end{array}$ & 106.3697 & 2283.7 & 14.5444 & 1.1791 & 0.6898 & 35 & 0.2979 & AVERAGE \\
\hline
\end{tabular}

\section{CONCLUSION}

In this work we have successfully colorized gray scale images of various sizes in $\mathrm{YCbCr}$ color space. Here we take a reference colored image or source image which is similar in texture to the target gray scale image, it is not necessary that this image is colorized version of the target gray scale image, it can be any similar colored image. The quality of output depends on the choice of this reference or source image. We convert both the target and source images in $\mathrm{YCbCr}$ decorrelated color space, to avoid cross channel artifacts, and divide the source image in windows of equal size. The size of window usually $7 \mathrm{X} 7$ suffices for all the images but may be increased for some problematic images. The properties of these windows are compared with those of target image based on texture features matching to find the best matching window. Then the luminance values of the pixels in this best matching window and gray scale image are compared and the chromatic properties of best matching pixel in source window are transferred to the corresponding gray scale image pixel

We compare our results with the output obtained by colorization using very popular technique of using only mean and standard deviation in $l \alpha \beta$ color space and come to the conclusion that the results obtained by colorization in $\mathrm{YCbCr}$ color space using our method are of better quality visually as well as on the basis of image quality parameters like Mean Square Error (MSE), Peak Signal to Noise Ratio (PSNR), Normalized Correlation (NK), Maximum Difference (MD), Normalized Absolute Error (NAE) etc. 


\section{REFERENCES}

\section{Journal Papers:}

[1] Daniel L. Ruderman, Thomas W. Cronin and Chuan-Chin Chiao, "Statistics of cone responses to natural images: implications for visual coding”, J. Opt. Soc. Am. A/ Vol. 15, pp. 8, August, 1998.

[2] Shaoyuan Sun, Jing Z., Liu G., Li Z., “Transfer Color to Night Vision Images.”, Chinese Optics Letters, Vol.3.(8), August, 2005.

[3] Ismail Acvibas, Kamoji Bulent, Sankur, and Khalid Sayood. "Statistical evaluation of image qualit" Journal of Electronic Imaging, 11(2):206-223, April 2002.

\section{Books:}

[4] Gonzalez R. C., Woods R. E., and Eddins, S. L. “digital image processing using matlab.” Pearson Education , New Delhi (India), Edition No. 1 (Reprint), 2004.

[5] Solomon C., Breckon T. "fundamentals of digital image processing.” Wiley - Blackwel, UK, Edition No. 1, 2011.

[6] D.R.J. Laming "sensory analysis" Academic Press, London, 1986.

\section{Proceedings Papers:}

[7] Semary N. A., "Texture recognition techniques for natural gray images", Radio Science Conférence, 2007. NRSC 2007. April, 2007.

[8] Smriti Kumar, Ayush Swarnkar, "Colorization of Gray Scale Images in l $\alpha$ Color Space using Mean and Standard Deviation" IEEE SCEECS 2012, MANIT, Bhopal (India), IEEE Conference Publications, March 2012.

[9] Weiss, Y., "Segmentation using eigenvectors: A unifying view", In Proc. IEEE Intl. Conf. Computer Vision, Vol. 2, Corfu, Greece, pp. 975-982, 1999

[10] Welsh, T., Ashikhmin, M. and Mueller, K., "Transferring color to grey scale images", In: Proceedings of 29th Annual Conference on Computer Graphics and Interactive Techniques, ACM-SIGGRAPH2002, pp. 277- 280, ACM Press, New York, USA, 2002. 